\title{
New Bounds on the Probability of a Finite Union of Events
}

\author{
Jun Yang, Fady Alajaji, Glen Takahara \\ Department of Mathematics and Statistics, Queen's University, Kingston, ON K7L3N6, Canada \\ E-mail: \{yangjun, fady, takahara\}@mast.queensu.ca
}

\begin{abstract}
The classes of all lower/upper bounds on the probability of a finite union of events which are expressed only in terms of the individual event probabilities and the sums of the pairwise event probabilities are considered. The optimal lower and upper bounds in each class are given numerically by solving a linear programming (LP) problem. Furthermore, a suboptimal analytical lower bound is established by solving a relaxed LP problem, which is at least as good as an existing bound due to Kuai, et al. [1]. Note that the new lower bounds can be further improved algorithmically by optimizing them over subsets [2], [3], and can be applied to general estimation problems involving the probability of a finite union. Finally, the new lower/upper bounds are illustrated by examining the symbol and bit error rates of an uncoded communication system used in conjunction with $M$ ary phase-shift keying (PSK) modulation over additive white Gaussian noise (AWGN) channels under maximum a posteriori (MAP) decoding.
\end{abstract}

\section{INTRODUCTION}

The lower/upper bound of $P\left(A_{1} \cup \cdots \cup A_{N}\right)$ in terms of the individual event probabilities $P\left(A_{i}\right)$ 's and the pairwise event probabilities $P\left(A_{i} \cap A_{j}\right)$ 's has been actively investigated in the recent past. The optimal bound can be obtained numerically by solving a linear programming (LP) problem with $2^{N}$ variables [4]. Since the number of variables is exponentially increasing with the number of events, $N$, some suboptimal numerical bounds are proposed, such as the bounds in [5], [6] using the dual basic feasible solutions to reduce the complexity of the LP problem, and the algorithmic Bonferroni-type upper and lower bounds in [7] [8].

Among the analytical bounds, the Kuai-Alajaji-Takahara bound (for convenience, hereafter refered to as the KAT bound) [1] is one of the analytical lower bounds that has been shown to be better than the Dawson-Sankoff bound [9] and D. de Caen's bound [10]. It is observed in [2], [3], [11] that the analytical bounds can be further improved algorithmically by optimizing over subsets. Furthermore, these general bounds can be applied to estimating the probability error in different coded or uncoded communication systems (e.g., see [2], [7], [8], [12]-[16]).

Note that the KAT bound which gives a lower bound on $P\left(A_{1} \cup \cdots \cup A_{N}\right)$ is expressed in terms of only individual event probabilities $\left\{P\left(A_{i}\right), i=1, \cdots, N\right\}$ and the sums of the pairwise event probabilities, i.e., $\left\{\sum_{j: j \neq i} P\left(A_{i} \cap A_{j}\right), i=\right.$ $1, \cdots, N\}$. Thus, knowledge of the individual pairwise event

This work was supported in part by NSERC of Canada. probabilities $P\left(A_{i} \cap A_{j}\right)$ is not required. In this paper, we revisit and investigate the same problem that bounds are established in terms of only $\left\{P\left(A_{i}\right), i=1, \cdots, N\right\}$ and $\left\{\sum_{j: j \neq i} P\left(A_{i} \cap A_{j}\right), i=1, \cdots, N\right\}$. Specifically, in the class of all lower/upper bounds that are expressed by only $P\left(A_{i}\right)$ 's and $\sum_{j} P\left(A_{i} \cap A_{j}\right)$ 's, the optimal lower and upper bounds are proposed numerically by solving an LP problem, which has only $N^{2}-N+1$ variables. Furthermore, a suboptimal analytical lower bound is proposed based on a relaxed LP problem, which is shown to be at least as good as the KAT bound. Due to space limitations, we refer to our journal paper [17] for the complete proofs.

Finally, we briefly illustrate the use of the new bounds by examining the symbol error rate (SER) and bit error rate (BER) of an uncoded communication system used in conjunction with $M$-ary phase-shift keying (PSK) modulation over additive white Gaussian noise (AWGN) channels under maximum $a$ posteriori (MAP) decoding. One should note that the proposed general bounds can be applied to other scenarios for estimating the probability error.

\section{LOWER/UPPER BOUNDS ON THE PROBABILITy OF A Finite UNION}

Consider a finite family of events $A_{1}, \cdots, A_{N}$ in a finite probability space $(\Omega, P)$, where $N$ is a fixed positive integer. For each $x \in \Omega$, let $p(x):=P(\{x\})$, and let the degree of $x$, denoted by $\operatorname{deg}(x)$, be the number of $A_{i}$ 's that contain $x$. Define

$$
a_{i}(k):=P\left(\left\{x \in A_{i}: \operatorname{deg}(x)=k\right\}\right),
$$

where $i=1, \cdots, N$ and $k=1, \cdots, N$. Then from [1, Lemma 1], we know that

$$
P\left(\bigcup_{i=1}^{N} A_{i}\right)=\sum_{i=1}^{N} \sum_{k=1}^{N} \frac{a_{i}(k)}{k} .
$$

Using the same notation as in [1], the lower bound is established only in terms of $\alpha_{i}:=P\left(A_{i}\right)$ and $\beta_{i}:=\sum_{j: j \neq i} P\left(A_{i} \cap\right.$ $\left.A_{j}\right), i=1, \cdots, N$. For simplicity, we denote $\gamma_{i}:=\alpha_{i}+\beta_{i}$. Then it is easy to verify that the following equalities hold:

$$
\sum_{k=1}^{N} a_{i}(k)=\alpha_{i} \text { and } \sum_{k=1}^{N} k a_{i}(k)=\gamma_{i}, \quad i=1, \cdots, N .
$$

In this paper, we consider the classes of lower/upper bounds that are established in terms of only $\alpha_{i}$ 's and $\gamma_{i}$ 's. 


\section{A. KAT Lower Bound}

The KAT lower bound which was derived in [1] can be denoted as follows.

$$
P\left(\bigcup_{i=1}^{N} A_{i}\right) \geq \sum_{i=1}^{N}\left\{\left[\frac{1}{\left\lfloor\frac{\gamma_{i}}{\alpha_{i}}\right\rfloor}-\frac{\frac{\gamma_{i}}{\alpha_{i}}-\left\lfloor\frac{\gamma_{i}}{\alpha_{i}}\right\rfloor}{\left(1+\left\lfloor\frac{\gamma_{i}}{\alpha_{i}}\right\rfloor\right)\left(\left\lfloor\frac{\gamma_{i}}{\alpha_{i}}\right\rfloor\right)}\right] \alpha_{i}\right\},
$$

where $\lfloor x\rfloor$ is the largest positive integer less than or equal to $x$. One should note that the KAT bound is expressed in terms of only the $P\left(A_{i}\right)$ 's and the $\sum_{j} P\left(A_{i} \cap A_{j}\right)$ 's, which means knowledge of the individual $P\left(A_{i} \cap A_{j}\right)$ is not required. Thus, the KAT bound is one example of a lower bound in the class of lower bounds we are interested in. The KAT bound was applied in [7] to bound the SER and BER for nonuniform signaling over AWGN channels, and optimized algorithmically in [2] to estimate the error rate of linear block codes.

\section{B. New Numerical Optimal Lower/Upper Bound}

Theorem 1: The optimal lower/upper bound of the classes of all lower/upper bounds that are established in terms of only $P\left(A_{i}\right)$ 's and $\sum_{j} P\left(A_{i} \cap A_{j}\right)$ 's are given by solving the following LPs:

$$
\begin{array}{ll}
\min _{\left\{a_{i}(k)\right\}} / \max _{\left\{a_{i}(k)\right\}} \sum_{i=1}^{N} \sum_{k=1}^{N} \frac{a_{i}(k)}{k} \\
\text { s.t. } \quad \sum_{k=1}^{N} a_{i}(k)=\alpha_{i}, \quad \sum_{k=1}^{N} k a_{i}(k)=\gamma_{i}, \quad i=1, \cdots, N, \\
& \sum_{i=1}^{N} a_{i}(k) \geq k a_{j}(k), \quad j=1, \cdots, N, \quad k=1, \cdots, N, \\
& a_{i}(k) \geq 0, \quad i=1, \cdots, N, \quad k=1, \cdots, N, \\
& \sum_{i=1}^{N} \sum_{k=1}^{N} \frac{a_{i}(k)}{k} \leq 1 .
\end{array}
$$

Proof: Without loss of generality, we only prove that the lower bound, $\ell_{\mathrm{OPT}}^{\prime}$ obtained via the minimization in (5) is optimal. The optimality of the upper bound can be proved similarly.

Let $\mathscr{L}$ denote the set of all lower bounds that are established in terms of only $\left\{\alpha_{i}, i=1, \cdots, N\right\}$ and $\left\{\gamma_{i}, i=1, \cdots, N\right\}$. Then any lower bound in $\mathscr{L}$, say $\ell^{\prime} \in \mathscr{L}$, is a function of only $\left\{\alpha_{i}\right\}$ 's and $\left\{\gamma_{i}\right\}$ 's. Also, to claim that $\ell^{\prime} \in \mathscr{L}$ is a lower bound for $P\left(A_{i} \cup A_{2} \cup \cdots \cup A_{N}\right)$, it means that for any events $\left\{A_{i}, i=1, \cdots, N\right\}$ that satisfy $P\left(A_{i}\right)=\alpha_{i}, i=1, \cdots, N$ and $\sum_{j} P\left(A_{i} \cap A_{j}\right)=\gamma_{i}, i=1, \cdots, N$, we must have $P\left(A_{1} \cup\right.$ $\left.A_{2} \cup \cdots \cup A_{N}\right) \geq \ell^{\prime}$. The optimal lower bound in class $\mathscr{L}$ is the lower bound $\ell_{\mathrm{OPT}} \in \mathscr{L}$ that satisfies $\ell_{\mathrm{OPT}} \geq \ell^{\prime}$ for all $\ell^{\prime} \in \mathscr{L}$.

First, we show that the last constraint of (5) and the additional constraint $\sum_{i=1}^{N} a_{i}(k) \geq k a_{j}(k)$ must hold for any family of events $\left\{A_{i}\right\}$, so that $\ell_{\mathrm{OPT}}^{\prime}$ is a lower bound. Note that the last constraint of (5) is just to ensure the upper bound is less than or equal to 1 , which can be relaxed for minimization problem. Also, it is easy to prove that for any $\left\{a_{i}(k)\right\}$ obtained by (1) from a family of events $\left\{A_{i}\right\}$, the additional constraints $\sum_{i=1}^{N} a_{i}(k) \geq k a_{j}(k)$ must hold for each $j=1, \cdots, N$ and $k=1, \cdots, N$. Therefore, $\ell_{\mathrm{OPT}}^{\prime}$ is a lower bound on $P\left(A_{1} \cup A_{2} \cup \cdots \cup A_{N}\right)$. Furthermore, since $\ell_{\mathrm{OPT}}^{\prime}$ is established in terms of only $\left\{\alpha_{i}\right\}$ 's and $\left\{\gamma_{i}\right\}$ 's, we have $\ell_{\mathrm{OPT}}^{\prime} \in \mathscr{L}$. Thus, we only need to prove $\ell_{\mathrm{OPT}}^{\prime} \geq \ell^{\prime}$ for all $\ell^{\prime} \in \mathscr{L}$. In the following, we first prove that $\ell_{\mathrm{OPT}}^{\prime}$ is achievable, i.e., a family of events $\left\{A_{i}\right\}$ can always be constructed which satisfies the constraints in (5) and satisfies $P\left(\bigcup_{i=1}^{N} A_{i}\right)=\ell_{\mathrm{OPT}}^{\prime}$. Then, we prove the optimality of $\ell_{\mathrm{OPT}}^{\prime}$, i.e., $\ell_{\mathrm{OPT}}^{\prime} \geq \ell^{\prime}$ for all $\ell^{\prime} \in \mathscr{L}$.

Achievability: We prove that for any $\left\{a_{i}(k)\right\}$ that satisfies the constraints of (5), it is always possible to construct a family of events $\left\{A_{i}\right\}$ such that $P\left(\left\{x \in A_{i}: \operatorname{deg}(x)=k\right\}\right)=a_{i}(k)$ holds. The construction method is given as follows:

- The set $\Omega$ is composed of $N \times N$ elementary outcomes, denoted as $\left\{x_{i}^{(k)}, i=1, \cdots, N, k=1, \cdots, N\right\}$. In the following, $\left\{x_{i}^{(k)}, i=1, \cdots, N\right\}$ are constructed separately for each $k$.

- Consider $N$ circles such that the $k$-th circle has a perimeter equals to $\sum_{i=1}^{N} \frac{a_{i}(k)}{k}, k=1, \cdots, N$. Then for the $k$-th circle, one should note that $\sum_{i=1}^{N} a_{i}(k)$ equals to $k$ times its perimeter. Furthermore, since $a_{j}(k) \leq \sum_{i=1}^{N} \frac{a_{i}(k)}{k}$ for all $j, a_{j}(k)$ is no larger than the perimeter of the $k$-th circle.

- For $j=1, \cdots, N$, we map the points on the arc of length $a_{j}(k)$ on the $k$-th circle from $2 \pi \frac{k \sum_{l=1}^{j-1} a_{l}(k)}{\sum_{i=1}^{N} a_{i}(k)}$ to $2 \pi \frac{k \sum_{l=1}^{j} a_{l}(k)}{\sum_{i=1}^{N} a_{i}(k)}$ to a set $B_{j}^{(k)}$. Then since for the $k$-th circle, $\sum_{i=1}^{N} a_{i}(k)$ equals to $k$ times its perimeter and $a_{j}(k)$ is no larger than its perimeter, it follows that every point on the $k$-th circle is mapped to exactly $k$ distinct sets in $\left\{B_{1}^{(k)}, \cdots, B_{N}^{(k)}\right\}$.

- On the $k$-th circle, the points at the following $N$ angles, $2 \pi\left(\frac{k \sum_{l=1}^{j} a_{l}(k)}{\sum_{i=1}^{N} a_{i}(k)}-\left\lfloor\frac{k \sum_{l=1}^{j} a_{l}(k)}{\sum_{i=1}^{N} a_{i}(k)}\right\rfloor\right), \quad j=1, \cdots, N$ divide the circle into (at most) $N$ arcs, and the points on each arc are mapped to the same $k$ sets in $\left\{B_{1}^{(k)}, \cdots, B_{N}^{(k)}\right\}$. Let $\left\{\theta_{j}^{(k)}, j=1, \cdots, N\right\}$ be the ordered tuple of

$\left\{2 \pi\left(\frac{k \sum_{l=1}^{j} a_{l}(k)}{\sum_{i=1}^{N} a_{i}(k)}-\left\lfloor\frac{k \sum_{l=1}^{j} a_{l}(k)}{\sum_{i=1}^{N} a_{i}(k)}\right\rfloor\right), j=1, \cdots, N\right\}$,

then $0=\theta_{1}^{(k)} \leq \theta_{2}^{(k)} \leq \cdots \leq \theta_{N}^{(k)} \leq 2 \pi$. Construct $x_{j}^{(k)}$ such that its probability $p\left(x_{j}^{(k)}\right)$ equals to the length of the $j$-th arc of the $k$-th circle, i.e.,

$$
p\left(x_{j}^{(k)}\right)= \begin{cases}\left(\theta_{j+1}^{(k)}-\theta_{j}^{(k)}\right) \frac{\sum_{i=1}^{N} a_{i}(k)}{2 \pi k} & \text { for } j<N, \\ \left(2 \pi-\theta_{N}^{(k)}\right) \frac{\sum_{i=1}^{N} a_{i}(k)}{2 \pi k} & \text { for } j=N .\end{cases}
$$

- Since the points on the $j$-th arc are mapped to $\left\{B_{i_{1 j}}^{(k)}, \cdots, B_{i_{k j}}^{(k)}\right\}$ where $\left\{i_{1 j}, \cdots, i_{k j}\right\} \in\{1, \cdots, N\}$ that contains $k$ different numbers, we let the elementary 
outcome $x_{j}^{(k)}$ belongs to $A_{i_{1 j}}, \cdots, A_{i_{k j}}$, respectively, i.e., $x_{j}^{(k)} \in A_{i_{1 j}} \cap \cdots \cap A_{i_{k j}}$.

- For each $k$, the total probability of all constructed outcomes equals to the perimeter of the circle, $\sum_{i=1}^{N} \frac{a_{i}(k)}{k}$. Also, each elementary outcome $x_{j}^{(k)}$ belongs to exactly $k$ events of $A_{1}, \cdots, A_{N}$. Finally, since there are in total $N \times N$ elementary outcomes $\left\{x_{j}^{(k)}, j=1, \cdots, N, k=\right.$ $1, \cdots, N\}$, each constructed $A_{i}$ contains a finite number of outcomes.

With the construction described above, it can be readily checked that the constructed $\left\{A_{i}\right\}$ satisfy $P\left(\left\{x \in A_{i}\right.\right.$ : $\operatorname{deg}(x)=k\})=a_{i}(k)$ for all $i=1, \cdots, N$. Since $\ell_{\text {OPT }}^{\prime}$ is achieved at one feasible point of (5), by the proposed construction method a family of events, say $\left\{A_{i}^{*}\right\}$, can be constructed so that $P\left(A_{1}^{*} \cup A_{2}^{*} \cup \cdots \cup A_{N}^{*}\right)=\ell_{\mathrm{OPT}}^{\prime}$. Since the first two constraints of (5) are also satisfied, we have $P\left(A_{i}^{*}\right)=\alpha_{i}$ and $\sum_{j} P\left(A_{i}^{*} \cap A_{j}^{*}\right)=\gamma_{i}$ for all $i$.

Optimality: For any $\ell^{\prime} \in \mathscr{L}$, by definition of $\mathscr{L}$ we have that $P\left(A_{1} \cup A_{2} \cup \cdots \cup A_{N}\right) \geq \ell^{\prime}$ for any events $\left\{A_{i}, i=1, \cdots, N\right\}$ satisfying $P\left(A_{i}\right)=\alpha_{i}, i=1, \cdots, N$ and $\sum_{j} P\left(A_{i} \cap A_{j}\right)=\gamma_{i}, i=1, \cdots, N$. Because the constructed family of events $\left\{A_{i}^{*}\right\}$ satisfies $P\left(A_{i}^{*}\right)=\alpha_{i}$ and $\sum_{j} P\left(A_{i}^{*} \cap A_{j}^{*}\right)=\gamma_{i}$ for all $i$, we must have $\ell_{\mathrm{OPT}}^{\prime}=$ $P\left(A_{1}^{*} \cup A_{2}^{*} \cup \cdots \cup A_{N}^{*}\right) \geq \ell^{\prime}$ for all $\ell^{\prime} \in \mathscr{L}$ as well.

The computational complexity of the new numerical lower/upper bound is low as the number of variables of the LP can be reduced to $N^{2}-N+1$ [17].

\section{New Analytical Lower Bound}

Another suboptimal analytical bound which is shown to be at least as good as the existing KAT bound can be proved in [17]. It is given as follows:

$P\left(\bigcup_{i=1}^{N} A_{i}\right) \geq \delta+\sum_{i=1}^{N}\left\{\left[\frac{1}{\chi\left(\frac{\gamma_{i}^{\prime}}{\alpha_{i}^{\prime}}\right)}-\frac{\frac{\gamma_{i}^{\prime}}{\alpha_{i}^{\prime}}-\chi\left(\frac{\gamma_{i}^{\prime}}{\alpha_{i}^{\prime}}\right)}{\left[1+\chi\left(\frac{\gamma_{i}^{\prime}}{\alpha_{i}^{\prime}}\right)\right]\left[\chi\left(\frac{\gamma_{i}^{\prime}}{\alpha_{i}^{\prime}}\right)\right]}\right] \alpha_{i}^{\prime}\right\}$,

where the function $\chi(\cdot)$ is defined by

$$
\chi(x)= \begin{cases}n-1 & \text { if } x=n \text { where } n \geq 2 \text { is a integer, } \\ \lfloor x\rfloor & \text { otherwise. }\end{cases}
$$

$\delta:=\left\{\max _{i}\left[\gamma_{i}-(N-1) \alpha_{i}\right]\right\}^{+} \geq 0, \alpha_{i}^{\prime}:=\alpha_{i}-\delta$, and $\gamma_{i}^{\prime}:=$ $\gamma_{i}-N \delta$.

Let $\ell_{\text {NEW }}$ denote the new analytical bound in (7) and $\ell_{\text {KAT }}$ denote the KAT lower bound (4). Then the improvement of the new analytical bound over the existing KAT bound, i.e., $\ell_{\mathrm{NEW}}-\ell_{\mathrm{KAT}}$, satisfies the following inequality (see also [17])

$$
\ell_{\mathrm{NEW}}-\ell_{\mathrm{KAT}} \geq\left\{\sum_{i=1}^{N} \frac{\left[N-\chi\left(\frac{\gamma_{i}}{\alpha_{i}}\right)\right]\left[N-\chi\left(\frac{\gamma_{i}}{\alpha_{i}}\right)-1\right]}{\chi\left(\frac{\gamma_{i}}{\alpha_{i}}\right)\left[\chi\left(\frac{\gamma_{i}}{\alpha_{i}}\right)+1\right]}\right\} \frac{\delta}{N} .
$$

\section{Numerical Examples of Lower/Upper Bounds}

We evaluate the lower/upper bounds using four numerical examples, shown in Tables I to IV, which are the same to the last four examples (System V to System VIII) in [17]. For
TABLE I

SYSTEM V.

\begin{tabular}{|c|c||c|c|c|}
\hline Outcomes $x_{i}$ & $p\left(x_{i}\right)$ & $A_{1}$ & $A_{2}$ & $A_{3}$ \\
\hline \hline$x_{0}$ & 0.145 & & & $\times$ \\
\hline$x_{1}$ & 0.045 & $\times$ & & $\times$ \\
\hline$x_{2}$ & 0.01 & $\times$ & $\times$ & $\times$ \\
\hline$x_{3}$ & 0.045 & $\times$ & $\times$ & \\
\hline$x_{4}$ & 0.145 & & $\times$ & \\
\hline
\end{tabular}

lower bounds, the results of the KAT bound, a recent bound by Feng, Li and Shen (FLS) [18] (this bound is an improvement of an earlier bound due to Gallot and Kounias), the new analytical bound (7) and the new numerical optimal bound (5) are shown in Table $\mathrm{V}$. We also include the algorithmic stepwise lower bound of [7], which is expressed by

$$
P\left(\bigcup_{i=1}^{N} A_{i}\right) \geq \sum_{i \in \zeta} P\left(A_{i}\right)-\sum_{i, j \in \zeta, i<j} P\left(A_{i} \cap A_{j}\right),
$$

where $\zeta$ is a subset of $\{1,2, \cdots, N\}$ which is determined by the Stepwise Algorithm [7].

For upper bounds, the new numerical optimal bound (5) and the union upper bound, i.e.

$$
P\left(\bigcup_{i=1}^{N} A_{i}\right) \leq \sum_{i=1}^{N} P\left(A_{i}\right)
$$

are also shown in Table $\mathrm{V}$. We also include the algorithmic Greedy upper bound [7], which can be written as

$$
P\left(\bigcup_{i=1}^{N} A_{i}\right) \leq \sum_{i=1}^{N} P\left(A_{i}\right)-\sum_{(i, j) \in T_{0}} P\left(A_{i} \cap A_{j}\right),
$$

where $T_{0}$ is a tree spanning the $N$ indices of the sets $A_{1}, \cdots, A_{N}$ and $(i, j)$ is an edge in $T_{0}$. The tree $T_{0}$ is obtained by the Greedy Algorithm [7].

From Table V, one can see that the new lower bounds (7) and (5) are sharper than the KAT bound in all examples. The new optimal upper bound agrees with the new optimal lower bound in the first two examples. Moreover, the FLS bound, which although requiring more information, is not guaranteed to be sharper than the new bounds and the KAT bounds. For example, the FLS bound is worse than the KAT bound in the first and fourth examples. It is better than the KAT bound but worse than the new lower bounds (7) and (5) in the second example, and better than the KAT lower bound and new lower bound (7) but worse than the new numerical bound (5) in the third example. Also, the new analytical lower bound (7) is sharper than the algorithmic stepwise lower bound (10) in System VI, and the numerical lower bound (5) is sharper than the stepwise lower bound (10) in Systems VI and VII. Finally, in all examples, the new numerical upper bound (5) agrees with the Greedy upper bound (12) and is much sharper than the union upper bound (11).

\section{Tight Bounds on Detection ERror Rates}

In this section, we briefly illustrate the new lower/upper bounds by estimating the $\operatorname{SER}\left(P_{s}\right)$ and the BER $\left(P_{b}\right)$ of 
TABLE V

COMPARISON OF LOWER BOUNDS.

\begin{tabular}{|c||c|c|c|c|c|c|c|c|c|}
\hline System & $P\left(\bigcup_{i=1}^{N} A_{i}\right)$ & KAT [1] & FLS [18] & New Lower (7) & Opt Lower (5) & Opt Upper (5) & Union (11) & Stepwise (10) & Greedy (12) \\
\hline \hline V & 0.3900 & 0.3833 & 0.3813 & 0.3900 & 0.3900 & 0.3900 & 0.5000 & 0.3900 & 0.3900 \\
\hline VI & 0.3252 & 0.2769 & 0.2972 & 0.3205 & 0.3252 & 0.3252 & 0.5612 & 0.3027 & 0.3252 \\
\hline VII & 0.5346 & 0.4434 & 0.4750 & 0.4562 & 0.5090 & 0.5346 & 0.8022 & 0.5009 & 0.5346 \\
\hline VIII & 0.5854 & 0.5412 & 0.5390 & 0.5464 & 0.5513 & 0.7070 & 1.5737 & 0.5673 & 0.7070 \\
\hline
\end{tabular}

TABLE II

SYSTEM VI.

\begin{tabular}{|c|c||c|c|c|c|}
\hline Outcomes $x_{i}$ & $p\left(x_{i}\right)$ & $A_{1}$ & $A_{2}$ & $A_{3}$ & $A_{4}$ \\
\hline \hline$x_{0}$ & 0.0962 & $\times$ & & & $\times$ \\
\hline$x_{1}$ & 0.0446 & & & & $\times$ \\
\hline$x_{2}$ & 0.0581 & & $\times$ & & $\times$ \\
\hline$x_{3}$ & 0.0225 & $\times$ & $\times$ & $\times$ & $\times$ \\
\hline$x_{4}$ & 0.0385 & $\times$ & & & \\
\hline$x_{5}$ & 0.0071 & $\times$ & & $\times$ & $\times$ \\
\hline$x_{6}$ & 0.0582 & & $\times$ & & \\
\hline
\end{tabular}

TABLE III

SYSTEM VII.

\begin{tabular}{|c|c||c|c|c|c|}
\hline Outcomes $x_{i}$ & $p\left(x_{i}\right)$ & $A_{1}$ & $A_{2}$ & $A_{3}$ & $A_{4}$ \\
\hline \hline$x_{0}$ & 0.1832 & $\times$ & & & \\
\hline$x_{1}$ & 0.1219 & & & $\times$ & \\
\hline$x_{2}$ & 0.0337 & $\times$ & $\times$ & $\times$ & $\times$ \\
\hline$x_{3}$ & 0.0256 & & $\times$ & $\times$ & \\
\hline$x_{4}$ & 0.0682 & & & & $\times$ \\
\hline$x_{5}$ & 0.0389 & & $\times$ & $\times$ & $\times$ \\
\hline$x_{6}$ & 0.0631 & & & $\times$ & $\times$ \\
\hline
\end{tabular}

nonuniform $M$-PSK modulated AWGN channels. Specifically, we consider a nonuniform i.i.d. binary source $\left\{X_{i}\right\}$, with distribution $P\{X=0\}=p$, that is transmitted via $M$ PSK modulation over an AWGN channel with single-sided power spectral density $N_{0}$. The source stream is grouped in blocks of $\log _{2} M$ bits which are each subsequently mapped to a modulation signal for transmission over the channel. We assume that $M$ is a power of 2 and Gray mapping is applied. At the receiver, optimal MAP decoding is performed in estimating the transmitted $M$-ary signal, which is known to be optimal in terms of minimizing the symbol error probability. Note that the exact SER and BER for nonuniform signaling can be obtained algorithmically as proposed in [19]. In [7], [16], the KAT bound, the FLS bound, the Stepwise Lower bound and the Greedy Upper bound are applied in the same

TABLE IV SYSTEM VIII.

\begin{tabular}{|c|c||c|c|c|c|}
\hline Outcomes $x_{i}$ & $p\left(x_{i}\right)$ & $A_{1}$ & $A_{2}$ & $A_{3}$ & $A_{4}$ \\
\hline \hline$x_{0}$ & 0.0330 & & & & $\times$ \\
\hline$x_{1}$ & 0.0705 & $\times$ & $\times$ & $\times$ & \\
\hline$x_{2}$ & 0.0876 & $\times$ & & $\times$ & $\times$ \\
\hline$x_{3}$ & 0.0608 & $\times$ & $\times$ & & $\times$ \\
\hline$x_{4}$ & 0.0865 & $\times$ & $\times$ & $\times$ & $\times$ \\
\hline$x_{5}$ & 0.0621 & & $\times$ & $\times$ & $\times$ \\
\hline$x_{6}$ & 0.0181 & & $\times$ & & \\
\hline$x_{7}$ & 0.0898 & $\times$ & & $\times$ & \\
\hline$x_{8}$ & 0.0770 & & $\times$ & $\times$ & \\
\hline
\end{tabular}

scenario to estimate the error probability.

\section{A. Average Symbol Error Rate}

Assume one of $M$ signals $s_{1}, \cdots, s_{M}$ is sent, $s_{k}$ is decoded by the MAP decoder if $P\left(s_{k} \mid r\right) \geq P\left(s_{i} \mid r\right), i=1, \cdots, M, i \neq$ $k$, where $r=s_{u}+n$ is the received signal and $n$ is Gaussian distributed noise with zero-mean and covariance matrix $\left(N_{0} / 2\right) \boldsymbol{I}$. The SER $P_{s}$ can be written as [7]:

$$
P_{s}=\sum_{u=1}^{M} P\left(\epsilon \mid s_{u}\right) P\left(s_{u}\right)=\sum_{u=1}^{M} P\left(\bigcup_{i \neq u} \epsilon_{i u}\right) P\left(s_{u}\right),
$$

where $P\left(\epsilon \mid s_{u}\right)$ is the conditional probability of error given that $s_{u}$ was sent, and $\epsilon_{i u}$ represents the event that $s_{i}$ has a higher MAP metric than $s_{u}$ given that $s_{u}$ was sent. Therefore, $P_{s}$ can be bounded by applying general lower/upper bounds to $P\left(\bigcup_{i \neq u} \epsilon_{i u}\right)$ in terms of $P\left(\epsilon_{i u}\right)$ and $P\left(\epsilon_{i u} \cap \epsilon_{j u}\right)$, which can be computed using the one-dimensional and two-dimensional Q-functions given in [7, Eq.(7)].

\section{B. Average Bit Error Rate}

Under the MAP decoding criterion, the BER $P_{b}$ can be written as [7] $P_{b}=\sum_{u=1}^{M} P_{b}(u) P\left(s_{u}\right)$ where

$$
P_{b}(u)=\frac{1}{\log _{2} M} \sum_{j=1}^{M} d\left(c_{j}, c_{u}\right) A_{j / u},
$$

and $A_{j / u}=1-P\left(\bigcup_{i \neq j} \epsilon_{i j u}\right)$ and $u=1, \cdots, M . c_{j}$ and $c_{u}$ are the bit assignments for signals $s_{j}$ and $s_{u}$, respectively. $d\left(c_{j}, c_{u}\right)$ is the Hamming distance between $c_{j}$ and $c_{u}$, and $\epsilon_{i j u}$ represents the event that symbol $s_{i}$ has a higher metric than symbol $s_{j}$ given that symbol $s_{u}$ was sent. As in the case for the symbol error probability, we can compute $P\left(\epsilon_{i j u}\right)$ and $P\left(\epsilon_{i j u} \cap \epsilon_{k j u}\right)$ by [7, Eq.(10) and Eq.(11)].

Therefore, $P_{b}$ can be bounded by applying the existing lower/upper bounds to $P\left(\bigcup_{i \neq j} \epsilon_{i j u}\right)$ in (14) so that an existing lower bound will result in an upper bound to $P_{b}$ and an existing upper bound will result in a lower bound to $P_{b}$.

\section{Numerical Results}

We illustrate the application of the new bounds by estimating the SER $P_{s}$ and BER $P_{b}$ for nonuniform signals with 8-PSK using Gray mapping over AWGN channels. The lower/upper bounds investigated include the KAT bound, the new analytical lower bound (7), the new numerical optimal lower/upper bounds (5), which are in terms of only $P\left(A_{i}\right)$ 's and $\sum_{j} P\left(A_{i} \cap A_{j}\right)$ 's. For comparison, we also include the 


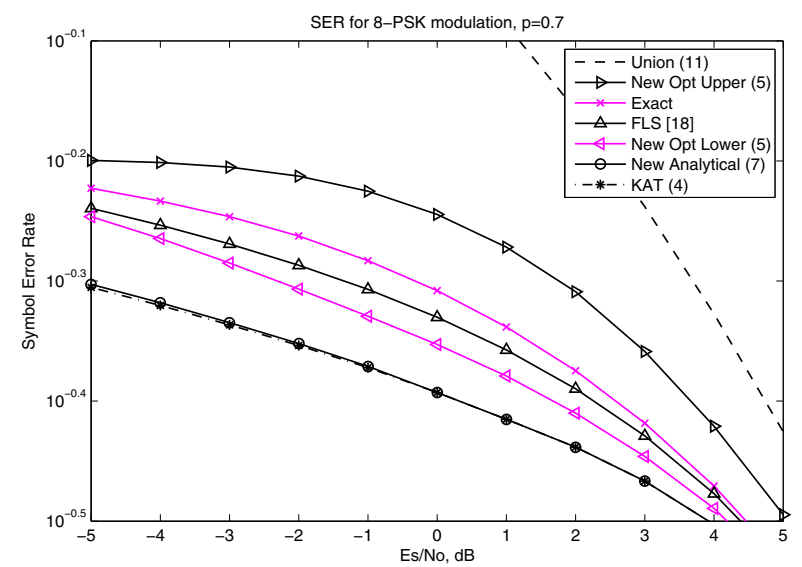

Fig. 1. SER for 8-PSK $(p=0.7)$

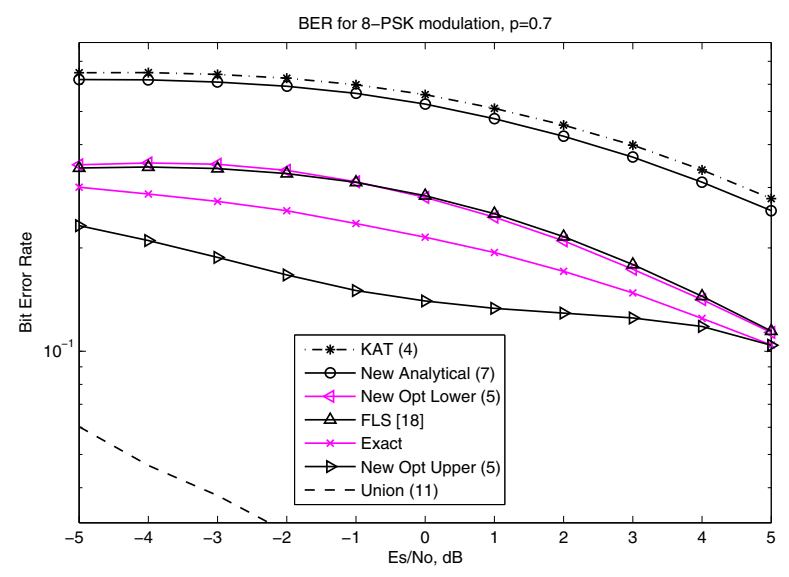

Fig. 2. BER for 8-PSK $(p=0.7)$

analytical FLS bound [18], which requires full knowledge of the pairwise event probabilities, and the union upper bound (11).

We first consider SER, $P_{s}$, for the nonuniform case $p=0.7$. The results for 8-PSK are shown in Fig. 1. From the figure, one can see that the new numerical lower/upper bounds give tight lower/upper bounds. Particularly, the numerical lower bound is much better than the KAT bound. Also, the new analytical bound performs slightly better than the existing KAT bound, as expected.

Finally, we estimate the BER for 8-PSK. The nonuniform case for $p=0.7$ is given in Fig. 2. One can remark that the new numerical lower bound is tighter than the FLS bound when the SNR is larger than $0 \mathrm{~dB}$, even though the FLS bound uses more information. Also, the new analytical lower bound is tighter than the existing KAT lower bound.

\section{CONCLUSION}

We present optimal numerical lower and upper bounds in the classes of all lower/upper bounds on the probability of a finite union of events, which are expressed in terms of the individual event probabilities and the sums of the pairwise event probabilities by solving an LP problem. We also establish a suboptimal analytical lower bound which is obtained by a relaxed LP problem, and is shown to be at least as good as the existing KAT bound [1]. The lower bounds can be further improved algorithmically via optimization over subset [2], [3] and all bounds can be applied to any general probability of error estimation problem, including channel coding. As an illustration, we apply the lower/upper bounds on a finite union of events to estimate the symbol error and bit error probabilities of an uncoded communication system with $M$-ary PSK, under MAP decoding.

\section{REFERENCES}

[1] H. Kuai, F. Alajaji, and G. Takahara, "A lower bound on the probability of a finite union of events," Discrete Mathematics, vol. 215, no. 1-3, pp. 147-158, 2000.

[2] F. Behnamfar, F. Alajaji, and T. Linder, "An efficient algorithmic lower bound for the error rate of linear block codes," IEEE Trans. Commun. vol. 55, no. 6, pp. 1093-1098, June 2007.

[3] F. M. Hoppe, "Improving probability bounds by optimization over subsets," Discrete Mathematics, vol. 306, no. 5, pp. 526-530, 2006.

[4] P. Veneziani, "Lower bounds of degree 2 for the probability of the union of N events via linear programming," June 2007, unpublished.

[5] A. Prékopa and L. Gao, "Bounding the probability of the union of events by aggregation and disaggregation in linear programs," Discrete Applied Mathematics, vol. 145, no. 3, pp. 444-454, 2005.

[6] B. Vizvári, "New upper bounds on the probability of events based on graph structures," Rutcor Research Report (Sep. 2004).

[7] H. Kuai, F. Alajaji, and G. Takahara, "Tight error bounds for nonuniform signaling over AWGN channels," IEEE Trans. Inf. Theory, vol. 46, no. 7, pp. 2712-2718, Nov. 2000.

[8] F. Behnamfar, F. Alajaji, and T. Linder, "Tight error bounds for spacetime orthogonal block codes under slow Rayleigh flat fading," IEEE Trans. Commun., vol. 53, no. 6, pp. 952-956, June 2005.

[9] D. Dawson and D. Sankoff, "An inequality for probabilities," Proceedings of the American Mathematical Society, vol. 18, no. 3, pp. 504-507, 1967.

[10] D. De Caen, "A lower bound on the probability of a union," Discrete Mathematics, vol. 169, no. 1, pp. 217-220, 1997.

[11] F. M. Hoppe, "The effect of redundancy on probability bounds," Discrete Mathematics, vol. 309, no. 1, pp. 123-127, 2009.

[12] G. Seguin, "A lower bound on the error probability for signals in white Gaussian noise," IEEE Trans. Inf. Theory, vol. 44, no. 7, pp. 3168-3175, Nov. 1998.

[13] S. Yousefi and A. K. Khandani, "A new upper bound on the ML decoding error probability of linear binary block codes in AWGN interference," IEEE Trans. Inf. Theory, vol. 50, no. 12, pp. 3026-3036, Dec. 2004.

[14] H. Nguyen and N. Tran, "Bonferroni-type bounds for CDMA systems with nonuniform signalling," IEEE Commun. Lett., vol. 9, no. 7, pp. 583-585, July 2005.

[15] R. Bettancourt, L. Szczecinski, and R. Feick, "BER evaluation of BICMID via Bonferroni-type bounds," IEEE Trans. Veh. Technol., vol. 57, no. 5, pp. 2815-2821, Sep. 2008.

[16] Z. Mao, J. Cheng, and J. Shen, "A new lower bound on error probability for nonuniform signals over AWGN channels," in IEEE Wireless Communications and Networking Conference (WCNC), 2013, pp. 30053009.

[17] J. Yang, F. Alajaji, and G. Takahara, "Lower bounds on the probability of a finite union of events," submitted to Discrete Mathematics. [Online]. Available: http://arxiv.org/abs/1401.5543

[18] C. Feng, L. Li, and J. Shen, "Some inequalities in functional analysis, combinatorics, and probability theory," The Electronic Journal of Combinatorics, vol. 17, no. R58, p. 1, 2010.

[19] L. Szczecinski, S. Aissa, C. Gonzalez, and M. Bacic, "Exact evaluation of bit- and symbol-error rates for arbitrary two-dimensional modulation and nonuniform signaling in AWGN channel," IEEE Trans. Commun., vol. 54, no. 5, pp. 1049-1056, June 2006. 